\title{
Comparing the Outcomes of Thyroid Surgical Procedures for Benign Diseases with Expertise of the Surgeon in a Tertiary Care Hospital in Rawalpindi, Pakistan
}

\author{
Nadir Mehmood ${ }^{1}$, Liaquat Ali Bhatti ${ }^{2}$, M. Idrees Anwar ${ }^{3}$, M. Aslam Chaudhry ${ }^{4}$, Malik Irfan Ahmed ${ }^{5}$, Maha \\ Nadir ${ }^{6}$, Hamza Nadir ${ }^{7}$, Mishal Fatima ${ }^{7}$ \\ ${ }^{1}$ Associate Professor, Department of Surgery, Holy Family Hospital, Rawalpindi, Punjab Pakistan \\ ${ }^{2}$ Associate Professor, Department of Surgery, Sir Ganga Ram Hospital, Lahore, Punjab Pakistan \\ ${ }^{3}$ Professor, Department of Surgery, Holy Family Hospital, Rawalpindi, Punjab Pakistan \\ ${ }^{4}$ Professor, Department of ENT, Benazir Bhutto Hospital, Rawalpindi, Punjab Pakistan \\ ${ }^{5}$ Senior Registrar, Department of Surgery, District Headquarter Hospital, Rawalpindi, Punjab Pakistan \\ ${ }^{6}$ House Officer, Department of Surgery, Rawalpindi Medical University, Rawalpindi, Punjab Pakistan \\ ${ }^{7}$ Medical Student, Rawalpindi Medical University, Rawalpindi, Punjab Pakistan
}

\begin{abstract}
Background: There is a lack of standardized guidelines regarding selection of appropriate thyroid surgery for patients with benign diseases. As a result, an inexperienced surgeon may select a more aggressive surgical option, which may increase the complication rate. The objective of this study was to compare the outcomes of thyroid surgical procedures for benign diseases with the expertise of the surgeon.

Material and Methods: A retrospective cross-sectional study from 1999 to 2018. The study setting is of a public sector tertiary care teaching hospital. Patients undergoing thyroid surgery (lobectomy with isthmusectomy, subtotal thyroidectomy (STT), near total thyroidectomy (NTT), or total thyroidectomy (TT)) were included. Expertise level 1,2 and 3 (L1, L2, L3) of the surgeon was based on years of experience or number of thyroid surgeries to their credit. Postoperative complications (hypocalcemia, recurrent laryngeal nerve (RLN) damage, airway obstruction, hemorrhage and mortality) were measured against type of thyroid surgery and expertise of the surgeon.

Results: A total of 833 thyroid surgeries were performed on 695 (83.43\%) females and 138 (16.57\%) males. About 502 (60.26\%) STT, 228 (27.37\%) TT, 61 (7.32\%) NTT, 42 (5.04\%) lobectomies with isthmusectomies were performed, with LI, 2 , and 3 surgeons performing $21.25 \%, 45.74 \%$ and $33 \%$ of these procedures, respectively. Surgeons with L1, 2 and 3 levels of expertise caused $49.47 \%, 33.45 \%$ and $17.08 \%$ of adverse events, respectively. Permanent hypocalcemia, RLN damage and mortality were significantly more common in surgeries performed by L1 compared with L2 and L3 surgeons $(\mathrm{P}<.05)$. Transient and permanent hypocalcemia, transient and permanent RLN damage and mortality were significantly more common for total thyroidectomy compared to subtotal thyroidectomy $(P<.01)$.

Conclusions: Minimizing the occurrence of complications like permanent hypocalcemia, RLN damage and mortality, expertise of the surgeon and anticipated difficulty of the procedure needs to be taken into account while selecting a thyroid procedure.

Key words: Hypocalcemia, Postoperative complications, Recurrent laryngeal nerve damage, Thyroidectomy.

Authors' Contribution:
'Conception; Literature research;
manuscript design and drafting; ${ }^{2,3}$ Critical
analysis and manuscript review; ${ }^{4-7}$ Data
analysis; Manuscript Editing.

$\begin{array}{ll}\text { Correspondence: } & \text { Article info: } \\ \text { Nadir Mehmood } & \text { Received: March 18, } 2021 \\ \text { Email: nadirmug@hotmail.com } & \text { Accepted: June 7, 2021 }\end{array}$

Accepted: June 7, 2021
\end{abstract}

Cite this article. Mehmood N, Bhatti LA, Anwar MI, Chaudhry MA, Ahmed MI, Nadir M, Fatima $M$. Comparing the outcomes of thyroid surgical procedures for benign diseases with expertise of the surgeon in a public hospital. J Islamabad Med Dental Coll. 2021; 10(2): 68-75. Doi: 10.35787/jimdc.v10i2.697
Funding Source: Nil

Conflict of Interest: Nil 


\section{Introduction}

Thyroid diseases are the one of the most common endocrine disorders throughout the world with varying prevalence rate of thyroid dysfunction. ${ }^{1}$ Thyroidectomy is a commonly performed procedure worldwide for the treatment of benign thyroid diseases ${ }^{2}$ and has low mortality and morbidity rates. ${ }^{3}$ It is performed by surgeons from various specialties and with varied experience. ${ }^{4}$ The outcome and complication rates are largely dependent on surgeon's skill and experience, the extent of surgery, indication of surgery, the nature of underlying disease and number of thyroid surgeries performed at that particular center as well as by the particular surgeon. ${ }^{5}$ Due to the advancements in surgical techniques, complication rate after thyroidectomy has decreased. In specialized centers, thyroidectomy has a very low complication rate and almost no mortality. ${ }^{6}$

Hypocalcemia of hypoparathyroidism is among the well-recognized complications of thyroid surgery and its incidence in literature varies widely depending on the type of procedure $(9 \%$ for total thyroidectomy), neck dissection, female gender and malignancy. ${ }^{7}$ Incidence of transient hypocalcemia has ranged from $1 \%$ to $68 \%$ and that of permanent hypocalcemia from $0 \%$ to $13 \% .^{8-11}$ Hypocalcemia is a sensitive measure of the quality of thyroid surgery. Direct injury, devascularization, or unintentional removal of parathyroid glands can result in hypocalcemia. ${ }^{12}$ As all the blood supply to the four parathyroid glands comes from inferior thyroid artery, it is logical inference that this procedure would increase postoperative hypocalcemia. Recurrent laryngeal nerve (RLN) palsy is a wellknown complication of thyroid surgery and its incidence is around $1-2 \% .{ }^{13}$ However, substantial recovery of vocal cord function can occur after thyroidectomy. ${ }^{14}$
Female sex, type of surgical procedure and changes in serum calcium perioperatively may be associated with hypocalcemia development. ${ }^{12}$ lodine deficient areas have a higher incidence of recurrent goiter which may require re-operation in a distorted anatomy due to fibrosis. ${ }^{15}$ So, there is an increased relative risk of recurrent laryngeal nerve, parathyroid and vascular injury in such patients. ${ }^{16}$ Postoperative neck hematoma formation may cause life threatening upper airway compromise, wound infection and scarring of the neck. New hemostatic devices have been developed that allow sutureless vessel ligation. ${ }^{17} \mathrm{~A}$ study performed to assess the safety of same day discharge of patients undergoing sutureless thyroidectomy using electrothermal bipolar vessel sealing system showed few major complications, reduced operative times and decreased incidence of hypocalcemia associated with this device. ${ }^{18}$

In the past, total thyroidectomy was usually performed for most thyroid cancers. Today, active surveillance or thyroid lobectomy/isthmusectomy can be employed for many patients with low-risk papillary thyroid cancers. ${ }^{19}$ Although total thyroidectomy as primary surgical management for thyroid disease has been in vogue in specialized units with low morbidity and mortality, ${ }^{6,20}$ there is fear of recurrence in the benign disease with initial conservative surgery (STT, NTT, lobectomy with isthmusectomy). This fear can be allayed by the fact that mean interval to re-operation for benign cases was 17.4 years and re-operative procedures comprised around $10 \%$ of thyroid surgery in a center over 20 years. ${ }^{21}$ Hence, re-operative procedures can be safely considered if performed by experienced surgeons. Because of widespread ambiguity on this matter and a gap in the literature that reviewed complications in general unspecialized units in 
resource starved countries, definitive recommendations are not available.

The lack of prospective randomized clinical trials for most management topics in thyroid surgery tends to make management recommendations, which are based on retrospective observations and often incomplete data, subject to selection biases that may be conflicting. Thus, the controversy continues as thyroid surgeons/experts review the same body of literature and reach differing conclusions based on their training, clinical perspective, experience, and personal bias.

Moreover, there was a mismatch between the international and local rates of post-operative complications in patients with benign thyroid disease. This prompted the managers and senior surgeons at our hospital to commence a study that assessed the rate of complication for thyroid surgery. The objective of this study was to compare the outcomes of thyroid surgical procedures for benign diseases with the expertise of the surgeon, and perform a review of national and international literature.

\section{Material and Methods}

This retrospective study spanning a period of 20 years (1999 to 2018) was carried out at Benazir Bhutto Hospital, a public sector tertiary-care teaching hospital affiliated with Rawalpindi Medical University, Rawalpindi, Punjab Pakistan. Approval for the study was obtained from the Rawalpindi Medical University Surgical Ethics Review Board, headed by the Dean of general surgery. Since this study utilized patient data from hospital and postoperative records, informed consent was not required. The STROBE (STrengthening the Reporting of OBservational studies in Epidemiology) cross sectional reporting guidelines was used while writing this report. ${ }^{22}$ Patient data was retrieved from the records of monthly surgical audits. Inclusion criteria comprised of all patients with goiter irrespective of age and sex who underwent any thyroid surgery (lobectomy with isthmusectomy, subtotal thyroidectomy (STT), near total thyroidectomy (NTT), or total thyroidectomy (TT)). Patients with malignant goiters, patients whose follow-up record for more than six months was not retrievable postoperatively or those with anesthetic or cardiovascular complications were excluded. Patients with history of prior or concomitant parathyroidectomy, known hyperparathyroidism, concomitant central neck lymph node dissection, preoperative hypocalcemia, or a history of previous head and neck related chemotherapy and/or radiotherapy were also excluded from this study.

As this was a retrospective study utilizing data from patient notes, no patient was directly involved in this study. Patients' records with detailed history, examination, thyroid function tests (T3, T4 \& TSH), radioactive thyroid scan, ultrasound examination and fine needle aspiration cytology (FNAC), CT/MRI scan were retrieved from the archives. Information regarding any preoperative vocal cord abnormality by indirect laryngoscopy was also obtained.

The detail of surgical option for each patient was retrieved from the operative notes. All thyroid surgeries were performed using standard surgical techniques. ${ }^{23}$ Surgical procedure carried out were thyroid lobectomy with isthmusectomy, subtotal, near total and total thyroidectomy, as according to empirical conviction of various heads of one surgical unit over 20 years. The level of expertise of the operating surgeon was divided in to three categories on either the number of total thyroid surgeries performed or years of surgical experience in a tertiary care teaching hospital. This data was acquired from their official service records and past five-year departmental records, respectively. Level 1 was a surgeon with more than five years of experience or 50 thyroid surgeries to his credit (junior assistant professor or a senior registrar); level 
2 , a surgeon with ten years of experience and 100 thyroid surgeries (associate or a senior assistant professor), and level 3 a senior surgeon with fifteen plus years of experience and 150 thyroid surgeries (professor or head of the department), respectively. The data for 23 operating surgeons during that period (1999-2018) is shown in Table I.
All the patient data were collected with a dedicated electronic Microsoft Office Excel Database (Microsoft Corp, Redmond, WA, USA). Differences between groups were analyzed with the Chi-square test. $P$-value less than .05 was considered significant.

\begin{tabular}{|l|c|c|c|c|c|c|c|}
\hline \multicolumn{7}{|c|}{ Table 1: Levels of expertise of operating surgeons from 1999 to 2018} \\
\hline Years & \multicolumn{2}{|c|}{ Level 1 (n) } & \multicolumn{2}{c|}{ Level 2 (n) } & \multicolumn{2}{|c|}{ Level $\mathbf{~ ( n ) ~}$} & Total (n) \\
\hline $1999-2003$ & SR (1) & AP (0) & SR (1) & AP (2) & Assoc/DS (1) & Prof (1) & 6 \\
\hline $1999-2003$ & SR (1) & AP (0) & SR 1 & AP (2) & Assoc/DS (1) & Prof (1) & 6 \\
\hline $2004-2010$ & SR (1) & AP (1) & SR 1 & AP (2) & AP/DS (1) & Prof (1) & 7 \\
\hline $2011-2018$ & SR (6) & AP (1) & SR 0 & AP (1) & AP (1) & Assoc (1) & 10 \\
\hline Total (n) & 8 & 2 & 2 & 5 & 3 & 3 & 23 \\
\hline
\end{tabular}

n-number; SR-Senior Registrar; AP-Assistant Professor; Assoc-Associate Professor; DS-District Surgeon; Prof-Professor

All the patients were observed post operatively for a variable period of time in the ward. The patients were followed in the outpatient at weekly interval for 2 weeks, monthly for 6 months and then yearly or as necessary according to the nature of the disease.

Medical records were retrieved from the monthly audit data. They were reviewed for variables like age, sex, preoperative diagnosis, extent of surgery, postoperative calcium levels, transient and permanent hypocalcemia, hoarseness, reason and need for tracheostomy, hemorrhage/ hematoma, any need to re-explore, wound infection, histopathological records and mortality. The postthyroidectomy hypocalcemia on first, second and fifth postoperative day and at six months following surgery was measured. Transient hypocalcemia was defined as serum calcium less than $8.0 \mathrm{mg} / \mathrm{dl}$ (2 $\mathrm{mmol} / \mathrm{L}$ ) on at least two consecutive measurements or signs and symptoms of hypocalcemia (perioral numbness, digital paresthesia, or positive Chvostek or Trousseau's sign). Permanent hypocalcemia was defined as the need for calcium and or vitamin $D$ supplements to maintain normal calcium levels at six months or more after the date of surgery.
The statistical analysis was performed using the IBM SPSS 23 software (Statistical Software, Chicago, IL, USA).

\section{Results}

Over a period of twenty years (199-2013), 833 thyroid patients were operated upon, of which 695 (83.43\%) were females and 138 (16.57\%) were males. The female-to-male ratio (F:M) was 5.04:1. The female-to-male patient distribution of surgical workload in OPD (43.3\% vs $56.7 \%)$, admitted cases (3.8\% vs $4.95 \%)$ and elective surgical procedures ( $47.5 \%$ vs $52.5 \%$ ) was almost equal (Table II). On an average, annually 41.65 thyroid procedures were performed, with a F:M of 5:1. About $90.5 \%$ of the females and $82.6 \%$ of males were euthyroid, while $87.6 \%$ females had multinodular goiter compared to 83.3\% males (Table III).

STT ( $n=502 ; 60.26 \%)$ were the most frequent thyroid surgeries performed, followed by $\pi \quad(n=228$; $27.37 \%)$, NTT ( $n=61 ; 7.32 \%)$ and lobectomy with isthmusectomy ( $n=42 ; 5.04 \%)$ with complications reported in 281 (33.73\%) cases (Table IV). 


\begin{tabular}{|l|r|r|c|c|c|c|c|c|c|c|c|}
\hline \multicolumn{8}{|c|}{ Table II: Frequency distribution of total OPD and admitted patients, elective procedures and thyroid surgeries in } \\
both male and female patients included in this retrospective study
\end{tabular}

OPD-Outpatient department; F-Female; M-Male; Ave-Average; \%-Percentage

\begin{tabular}{|l|c|c|}
\hline \multicolumn{3}{|c|}{$\begin{array}{c}\text { Table III: Gender wise distribution of clinical } \\
\text { presentation of patients with thyroid lesions }\end{array}$} \\
\hline $\begin{array}{l}\text { Clinical } \\
\text { Presentation }\end{array}$ & $\begin{array}{c}\text { Male } \\
\mathbf{n}(\%)\end{array}$ & $\begin{array}{c}\text { Female } \\
\mathbf{n}(\%)\end{array}$ \\
\hline Euthyroid & $114(82.6)$ & $629(90.5)$ \\
\hline Hyperthyroid & $19(13.8)$ & $43(6.2)$ \\
\hline Hypothyroid & $5(3.6)$ & $23(3.3)$ \\
\hline MNG & $115(83.3)$ & $609(87.6)$ \\
\hline $\begin{array}{l}\text { Solitary } \\
\text { Nodule }\end{array}$ & $14(10.1)$ & $49(7)$ \\
\hline Diffuse & $9(6.5)$ & $37(5.3)$ \\
\hline Total & 138 & 695 \\
\hline
\end{tabular}

MNG-Multinodular goiter

LI, 2, and 3 surgeons performed $21.25 \%, 45.74 \%$ and $33 \%$ of thyroid surgeries, respectively (Table IV \& V). Regarding complications occurring in thyroid procedures, L1 surgeons were responsible for $49.47 \%$ of adverse events, L2 for $33.45 \%$ and L3 for
17.08\%, respectively (Table V). Complications like permanent hypocalcemia, recurrent laryngeal nerve damage and mortality were significantly more common in surgeries performed by L1 surgeons as compared to surgeons with L2 and L3 level of expertise $(P<.05) \quad$ (Table $V)$. Transient and permanent hypocalcemia, transient and permanent RLN damage and mortality were significantly more common for total thyroidectomy compared to subtotal thyroidectomy $(P<.01)$. The complication rate of total thyroidectomy for permanent hypocalcemia, permanent RLN paralysis and mortality was $7.9 \%, 6.1 \%$ and $7.9 \%$ respectively for all levels of expertise (Table V). The definitive complication rate (combined rates for permanent hypocalcemia, permanent RLN paralysis and mortality) was $36.36 \%$ for $L 1,14.7 \%$ for $L 2$ and $7.3 \%$ for L3, respectively (Table V).

\begin{tabular}{|l|c|c|c|c|}
\hline \multicolumn{5}{|c|}{ Table IV: Distribution of type of thyroid surgeries, complications and level of surgical expertise in the study } \\
population
\end{tabular}

STT-Sub-total thyroidectomy; NTT-Near-total thyroidectomy; TT-Total thyroidectomy 


\begin{tabular}{|c|c|c|c|c|c|c|c|c|}
\hline \multirow[b]{2}{*}{$\begin{array}{l}\text { Type of } \\
\text { Surgery }\end{array}$} & \multirow[b]{2}{*}{$\begin{array}{l}\text { Levels of } \\
\text { Expertise }\end{array}$} & \multicolumn{7}{|c|}{ Complications } \\
\hline & & $\begin{array}{c}\text { Transient } \\
\text { Hypocalcemia }\end{array}$ & $\begin{array}{c}\text { Permanent } \\
\text { Hypocalcemia }\end{array}$ & $\begin{array}{c}\text { Transient } \\
\text { RLN } \\
\text { Paralysis }\end{array}$ & $\begin{array}{c}\text { Permanent } \\
\text { RLN } \\
\text { Paralysis }\end{array}$ & Tracheostomy & Hemorrhage & Mortality \\
\hline \multirow{5}{*}{$\begin{array}{l}\text { TT } \\
(n=228)\end{array}$} & L1 (n=99) & $13(13 \%)$ & $14(14 \%)$ & $12(12 \%)$ & $10(10 \%)$ & $9(9 \%)$ & $1(1 \%)$ & $12(12 \%)$ \\
\hline & $\mathrm{L} 2(\mathrm{n}=61)$ & $17(28 \%)$ & $3(5 \%)$ & $10(16 \%)$ & $3(5 \%)$ & $2(3.2 \%)$ & 0 & $3(5 \%)$ \\
\hline & L3 $(n=68)$ & $11(16 \%)$ & $1(1.47 \%)$ & $4(5.8 \%)$ & $1(1.47 \%)$ & $2(3.2 \%)$ & $1(1 \%)$ & $3(4.4 \%)$ \\
\hline & Percentage & 18 & 7.9 & 11.4 & 6.1 & 5.7 & 0.87 & 7.9 \\
\hline & $P$-value & $>.05$ & $<.01$ & $>.05$ & $<.05$ & $>.05$ & $>.05$ & $<.05$ \\
\hline \multirow{5}{*}{$\begin{array}{l}\text { Other } \\
\text { surgeries } \\
(n=605)\end{array}$} & $\mathrm{L} 1(\mathrm{n}=78)$ & 13 & 2 & 12 & 1 & 2 & 4 & 3 \\
\hline & $\mathrm{L} 2(\mathrm{n}=320)$ & 8 & 0 & 8 & 0 & 2 & 4 & 1 \\
\hline & L3 $(n=207)$ & 5 & 0 & 2 & 0 & 0 & 0 & 0 \\
\hline & Percentage & 4.3 & 0.3 & 3.6 & 0.1 & 0.6 & 1.3 & 0.6 \\
\hline & $P$-value & $<.05$ & $<.05$ & $<.05$ & $>.05$ & $>.05$ & $<.05$ & $<.05$ \\
\hline
\end{tabular}

TT- Total Thyroidectomies; RLN-Recurrent laryngeal nerve

\section{Discussion}

The results of our study suggest that rates of complication are significantly higher for total thyroidectomies performed for any benign condition and also for surgeons with lower level of expertise. Literature suggests that it is possible to achieve a definitive complication rate approaching $0 \%$ for total thyroidectomy performed for $\mathrm{MNG}^{24}$ whereas definitive complication rate of total thyroidectomy in our study, even for a level 3 surgeon, was around $7 \%$. This can be due to the unspecialized and general training of our surgeons. Another study comparing expertise of surgeons with complication rates for thyroidectomies found an overall complication rate of $5.1 \%$ for surgeons who had performed more than 100 thyroidectomies (all types) in a 6-year period which was much lower compared to other surgeons with lesser expertise. ${ }^{25}$ One study concluded that the complication rate after thyroidectomy can be significantly reduced if the procedure is performed by an experienced surgeon. ${ }^{6}$ Our study also shows a significant association between level of expertise and complication rate but the complication rates are higher for all types of thyroidectomies when compared to similar studies performed in specialized units in other parts of the world..$^{24,25}$

Literature review indicates higher complication rates for total thyroidectomy like postoperative hypoparathyroidism (about $6 \%$ ), recurrent laryngeal nerve paralysis and wound complications $(\approx 1 \%) .{ }^{26,27}$ Complications in total thyroidectomies were also higher in our study as compared to other surgeries but a worrying sign was the presence of higher complication rates for other surgeries as well. Lack of experience on behalf of the surgeon performing total thyroidectomy can explain the higher morbidity and increased complication rates, but higher complications in other thyroid surgeries point towards a systemic workload and staff mismatch problem. There was a progressive increase in number of thyroid surgeries over 20 years along with an increase in outpatient workload, admissions and other elective procedures (Table II). In contrast, the number of operating surgeons increased from 6 in 1998-2003 to only 10 in 2011-2018 while the number of Level 3 surgeons remained constant (Table I).

This indicates an exponential increase in workload for the surgeons at all levels. It is, therefore, not 
surprising that complications increase when L1 surgeons are obligated to take on operating duties, perhaps, with inadequate experience. Another issue is the trend of changing practices with the changing leadership. A new department head, oftentimes, promotes a certain experience-based practice. So, where his/her predecessor was in favor of STT, the new head might promote TT or NTT to prevent recurrence. These changing practices do not take into account the level of expertise of the junior surgeons which leads to more complications. The surgeons either do not select the correct procedure for the patient according to the pathology or their perceived level of expertise is inadequate in avoiding complications. Certain strategies can be implemented to minimize these risk factors. Preoperatively, a thyroidectomy difficulty scale can be employed ${ }^{28}$ and a detailed management plan and type of surgery needed to be performed should be selected based on this. It is prudent for a more experienced member of the team to perform the surgery if complications are anticipated and the procedure selected must take into account all the available evidence. In order to achieve this, surgeons should receive appropriate training and follow best practice guidelines.

This study included data from over a period of 20 years and thus its findings and interpretations are reflective of the culture of surgical practice in a tertiary care teaching hospital with resource constraints. The surgical procedures performed for a given indication often reflect the surgeon's or the department head's convictions based on his/her past experience and are not always completely evidence-based. This is a limitation for many surgical studies that are performed.

Since, the study is set in a public sector hospital catering to patients from underprivileged backgrounds from far flung areas, follow-up could not be ensured in all cases and the findings in this study may be underreporting the actual occurrence of complications.

\section{Conclusion}

Our study concluded that to minimize the rate of definitive complications, expertise of the surgeon and anticipated difficulty of the procedure needs to be taken into account. However, these outcomes can only be achieved if the surgeon is well-versed in the anatomy of the gland, is mindful of its pathology, and has received proper training.

\section{References}

1. Maniakas A, Davies L, Zafereo ME. Thyroid Disease Around the World. Otolaryngol Clin North Am. 2018; 51: 631-642. Doi: 10.1016/j.otc.2018.01.014.

2. Makay Ö. Less than total thyroidectomy for goiter: When and how? Gland Surg. 2017; 6(Suppl 1): S49S58. Doi: 10.21037/gs.2017.10.02.

3. Padur AA, Kumar N, Guru A, Badagabettu SN, Shanthakumar SR, Virupakshamurthy $M B$, et al. Safety and effectiveness of total thyroidectomy and its comparison with subtotal thyroidectomy and other thyroid surgeries: a systematic review. J Thyroid Res. 2016; 2016: 1-6. Doi: $10.1155 / 2016 / 7594615$.

4. Khanzada TW, Samad A, Memon W, Kumar B. Post thyroidectomy complications: the Hyderabad experience. J Ayub Med Coll. 2010; 22(1): 65-8. PMID: 21409907.

5. Meltzer C, Hull M, Sundang A, Adams JL. Association Between Annual Surgeon Total Thyroidectomy Volume and Transient and Permanent Complications. JAMA Otolaryngol Head Neck Surg. 2019; 145(9): 830-7. Doi: 10.1001/jamaoto.2019.1752.

6. Ahmad Z, Kutaiman A, Hassan Y, Amin P, Khan M. Complication rates of thyroidectomy by an experienced, high-volume thyroid surgeon in a private hospital in Abu Dhabi, United Arab Emirates. Hamdan Med J. 2018; 11(1): 17-21. Doi: 10.7707/hmj.735.

7. Baldassarre RL, Chang DC, Brumund KT, Bouvet M. Predictors of hypocalcemia after thyroidectomy: results from the nationwide inpatient sample. ISRN Surg. 2012; 838614. Doi: 10.5402/2012/838614.

8. Eismontas V, Slepavicius A, Janusonis V, Zeromskas $P$, Beisa V, Strupas K, et al. Predictors of postoperative hypocalcemia occurring after a total thyroidectomy: 
results of prospective multicenter study. BMC Surg. 2018; 18(1): 55. Doi: 10.1186/s12893-018-0387-2.

9. Merchavy S, Marom T, Forest VI, Hier M, Mlynarek A, McHugh $\mathrm{T}$, et al. Comparison of the incidence of postoperative hypocalcemia following total thyroidectomy vs completion thyroidectomy. Otolaryngol Head Neck Surg. 2015; 152(1): 53-6.

10. Doi: $10.1177 / 0194599814556250$.

11. Edafe $O$, Balasubramanian SP. Incidence, prevalence and risk factors for post-surgical hypocalcaemia and hypoparathyroidism. Gland Surg. 2017; 6(Suppl 1): S59-S68. Doi: 10.21037/gs.2017.09.03.

12. Baloch N, Taj S, Anwer M, Naseem M. Frequency of hypocalcaemia following total thyroidectomy. Pak J Med Sci. 2019; 35(1): 262-5. Doi: 10.12669/pjms.35.1.93.

13. Del Rio P, Rossini M, Montana CM, Viani L, Pedrazzi G, Loderer $T$, et al. Postoperative hypocalcemia: analysis of factors influencing early hypocalcemia development following thyroid surgery. BMC Surg. 2019; 18(S1): 25. Doi: 10.1186/s12893-019-0483-y.

14. Gambardella C, Polistena A, Sanguinetti A, Patrone R, Napolitano S, Esposito D, et al. Unintentional recurrent laryngeal nerve injuries following thyroidectomy: Is it the surgeon who pays the bill? Int J Surg. 2017; 41(1): S55-9. Doi: 10.1016/j.ijsu.2017.01.112.

15. Pantvaidya G, Mishra A, Deshmukh A, Pai PS, D'Cruz $A$. Does the recurrent laryngeal nerve recover function after initial dysfunction in patients undergoing thyroidectomy? RLN recovery after thyroidectomy. Laryngoscope Investig Otolaryngol. 2018; 3(3): 249-52. Doi: 10.1002/lio2.167.

16. Gurleyik E, Cetin F, Dogan S, Yekenkurul E, Onsal U, Gursoy F, Ipor A. Displacement of the Recurrent Laryngeal Nerve in Patients with Recurrent Goiter Undergoing Redo Thyroid Surgery. J Thyroid Res. 2018; 4763712. Doi: 10.1155/2018/4763712.

17. Levin KE, Clark AH, Duh QY, Demeure M, Siperstein $A E$, Clark $\mathrm{OH}$. Reoperative thyroid surgery. Surgery. 1992; 111(6): 604-9. PMID: 1595056.

18. Kiriakopoulos A, Dimitrios T, Dimitrios L. Use of a Diathermy System in Thyroid Surgery. Arch Surg. 2004; 139(9): 997-1000. Doi: 10.1001/archsurg.139.9.997.

19. Inabnet WB, Shifrin A, Ahmed L, Sinha P. Safety of Same Day Discharge in Patients Undergoing Sutureless Thyroidectomy: A Comparison of Local and General Anesthesia. Thyroid. 2007; 18(1): 57-61. Doi: 10.1089/thy.2007.0148.
20. Tuttle RM, Zhang L, Shaha A. A clinical framework to facilitate selection of patients with differentiated thyroid cancer for active surveillance or less aggressive initial surgical management. Expert Rev Endocrinol Metab. 2018; 13(2): 77-85. Doi: 10.1080/17446651.2018.1449641.

21. Hu J, Zhao N, Kong R, Wang D, Sun B, Wu L. Total thyroidectomy as primary surgical management for thyroid disease: surgical therapy experience from 5559 thyroidectomies in a less-developed region. World J Surg Oncol. 2016; 14(1): 20. Doi: 10.1186/s12957-016-0772-1.

22. Hardman JC, Smith JA, Nankivell P, Sharma N, Watkinson JC. Re-operative thyroid surgery: a 20year prospective cohort study at a tertiary referral centre. Eur Arch Otorhinolaryngol. 2015; 272(6): 1503-8. Doi: 10.1007/s00405-014-3068-5.

23. von Elm E, Altman DG, Egger M, Pocock SJ, Gøtzsche PC, Vandenbroucke JP. Strengthening the Reporting of Observational Studies in Epidemiology (STROBE) statement: guidelines for reporting observational studies. BMJ. 2007; 335(7624): 806-8. Doi: 10.1136/bmj.39335.541782.AD.

24. Farquharson MM, Brendan. Surgery of the neck. Farquharson's Textbook of Operative General Surgery. 10th ed: CRC Press; 2014. p. 155-78.

25. Ciftci F, Sakalli E, Abdurrahman I. Total versus bilateral subtotal thyroidectomy for benign multinodular goiter. Int J Clin Exp Med. 2015; 8(3): 4596600. PMID: 26064391.

26. Sosa JA, Bowman HM, Tielsch JM, Powe NR, Gordon TA, Udelsman $R$. The importance of surgeon experience for clinical and economic outcomes from thyroidectomy. Ann Surg. 1998; 228(3): 320-30. Doi: 10.1097/00000658-199809000-00005.

27. Bhattacharyya N, Fried MP. Assessment of the Morbidity and Complications of Total Thyroidectomy. Arch Otolaryngol Head Neck Surg. 2002; 128(4): 389-92. Doi: 10.1001/archotol.128.4.389.

28. Alqahtani $S M$, Almussallam $B$, Alatawi $A$, Alsuhaimi NA, Albalawi A, Albalawi NS, et al. PostThyroidectomy Complications and Risk Factors in Tabuk, Saudi Arabia: A Retrospective Cohort Study. Cureus. 2020; 12(10): e10852. doi:10.7759/cureus.10852.

29. Bothra S, Sabaretnam M, Kannujia A, Chand G, Agarwal G, Mishra SK, et al. Patient, thyroid, and surgeon related factors that make thyroidectomy difficult-cohort study. Ann Med Surg. 2020; 49: 14-8. Doi: 10.1016/j.amsu.2019.11.010. 